\title{
Determination of minimal realisation of one-dimensional continuous-time fractional linear system
}

\author{
Konrad Andrzej Markowski ${ }^{1}$
}

Received: 6 December 2015 / Revised: 21 January 2016 / Accepted: 10 February 2016 / Published online: 19 February 2016

(C) The Author(s) 2016. This article is published with open access at Springerlink.com

\begin{abstract}
In this paper, a new alternative method for the determination of the set of all minimal positive and non-positive realisations of the one-dimensional fractional continuous-time linear systems based on the one-dimensional digraphs theory has been presented. In addition, all realisations in the set are minimal. For the proposed method, an algorithm was also constructed. The algorithm is based on a parallel computing method to gain needed speed and computational power for such a solution. The proposed method is discussed and illustrated with numerical examples.
\end{abstract}

Keywords Fractional - Minimal realisation - Positive system · Digraphs · Algorithm · Continuous-time

Mathematics Subject Classification 26A33 and 05C20 . $15 \mathrm{~B} 48 \cdot 26 \mathrm{C} 15 \cdot 65 \mathrm{Y} 04$

\section{Introduction}

In the recent years, many researchers have been interested in positive and non-positive linear systems. Examples of positive systems are industrial processes involving chemical reactors, heat exchangers and distillation columns, storage

Research has been financed with the funds of the Statutory Research of 2016.

\footnotetext{
Konrad Andrzej Markowski

Konrad.Markowski@ee.pw.edu.pl

1 Faculty of Electrical Engineering, Institute of Control and Industrial Electronics, Warsaw University of Technology, Koszykowa 75, 00-662 Warsaw, Poland
}

systems, compartmental systems, water and atmospheric pollution models. A variety of models having positive linear systems behavior can be found in engineering, management science, economics, social sciences, biology and medicine, etc. In positive systems, inputs, state variables and outputs take only non-negative values. Positive linear systems are defined on cones and not on linear spaces. Therefore, the theory of positive systems is more complicated than standard systems [1-4]. The realisation problem is a very difficult task [1]. In many research studies, we can find a canonical form of the system, i.e. constant matrix form, which satisfies the system described by the transfer function [2,5]. With the use of this form, we are able to write only one realisation of the system, while there exists a set of solutions. The state of the art in a positive systems theory is given in the monographs [2, 4, 6]. In [7-9] a solution for finding a set of possible realisations of the characteristic polynomial was proposed, and in [10-12] a solution of the positive fractional realisation problem based on digraph theory was presented. This means that we can find many sets of matrices which fit into a system transfer function.

The first definition of the fractional derivative was introduced by Liouville and Riemann at the end of the nineteenth century [13]. Mathematical fundamentals of fractional calculus are given in the monographs [13-18]. Some others applications of fractional-order systems can be found in [19-27].

The main purpose of this paper is to present a method for determination of a minimal realisation of the onedimensional continuous-time fractional linear system of a given proper transfer function. Proposed method is based on the digraph-theory. Conditions for the existence of a minimal realisation of a given transfer function will be established, and an algorithm for computation of a minimal realisation will be proposed. This work has been organ- 
ised as follows: Sect. 2 presents some notations and basic definitions of a digraph theory. In Sect. 3, we present basic properties of the theory fractional order system. In this section, fractional continuous-time system is defined as the state-space representation, and the problem is formulated. Then, in Sect. 4, we construct an algorithm for a determination of positive and non-positive minimal realisations of the fractional continuous-time system. Finally, we demonstrate some numerical examples (Sect. 5), present concluding remarks, open problems and bibliography positions.

\section{Notion and basic definitions of digraphs theory}

\subsection{Notion}

In this paper, the following notion will be used. The matrices will be denoted by the bold font (for example $\mathbf{A}, \mathbf{B}, \ldots$ ), the sets by the double line (for example $\mathbb{A}, \mathbb{B}, \ldots$ ), lower/upper indices and polynomial coefficients will be written as a small font (for example $a, b, \ldots$ ), digraphs will be denoted using a mathcal font $\mathscr{D}$ and differentiation operator will be denoted using mathfrak font $\mathfrak{D}$.

The set $n \times m$ real matrices will be denoted by $\mathbb{R}^{n \times m}$ and $\mathbb{R}^{n}=\mathbb{R}^{n \times 1}$. If $\mathbf{G}=\left[g_{i j}\right]$ is a matrix, we write $\mathbf{G} \gg 0$ (matrix $\mathbf{G}$ is called strictly positive), if $g_{i j}>0$ for all $i, j$; $\mathbf{G}>0$ (matrix $\mathbf{G}$ is called positive), if $g_{i j}>0$ for all $i, j$; $\mathbf{G} \geqslant 0$ (matrix $\mathbf{G}$ is called non-negative), if $g_{i j} \geqslant 0$ for all $i$, $j$. The set of $n \times m$ real matrices with non-negative entries will be denoted by $\mathbb{R}_{+}^{n \times m}$ and $\mathbb{R}_{+}^{n}=\mathbb{R}_{+}^{n \times 1}$. The set of $n \times n$ real matrices with off-diagonal non-negative entries will be denoted by $\mathbb{M}^{n \times n}$ and the set of positive integers will be denoted by $\mathbb{Z}_{+}$. The $n \times n$ identity matrix will be denoted by $\mathbf{I}_{n}$. For more information about matrix theory, an interested reader is referred, for instance, to: $[28,29]$.

\subsection{Basic definitions of digraphs theory}

A directed graph (or just digraph) $\mathscr{D}$ consists of a non-empty finite set $\mathbb{V}(\mathscr{D})$ of elements called vertices and a finite set $\mathbb{A}(\mathscr{D})$ of ordered pairs of distinct vertices called arcs [30]. We call $\mathbb{V}(\mathscr{D})$ the vertex set and $\mathbb{A}(\mathscr{D})$ the arc set of $\mathscr{D}$. We will often write $\mathscr{D}=(\mathbb{V}, \mathbb{A})$ which means that $\mathbb{V}$ and $\mathbb{A}$ are the vertex set and arc set of $\mathscr{D}$, respectively. The order of $\mathscr{D}$ is the number of vertices in $\mathscr{D}$. The size of $\mathscr{D}$ is the number of arc in $\mathscr{D}$. For an $\operatorname{arc}\left(v_{1}, v_{2}\right)$ the first vertex $v_{1}$ is its tail and the second vertex $v_{2}$ is its head.

There exists $\mathscr{A}$-arc from vertex $v_{j}$ to vertex $v_{i}$ if and only if the $(i, j)$ th entry of the matrix $\mathbf{A}$ is non-zero for $i, j \in \mathbb{Z}_{+}$. There exists $\mathscr{B}$-arc from source $s_{l}$ to vertex $v_{j}$, if and only if the $l$ th entry of the matrix $\mathbf{B}$ is non-zero for $j, l \in \mathbb{Z}_{+}$.

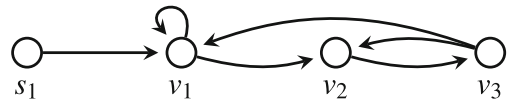

Fig. 1 One-dimensional digraph

Consider the following example. Let be given the positive system single input described by the following matrices

$(\mathbf{A}, \mathbf{B})=\left(\left[\begin{array}{lll}1 & 0 & 1 \\ 1 & 0 & 1 \\ 0 & 1 & 0\end{array}\right],\left[\begin{array}{l}1 \\ 0 \\ 0\end{array}\right]\right)$

we can draw one-dimensional digraph $\mathscr{D}^{(1)}$ (see Fig. 1), consisting of vertices $v_{1}, v_{2}, v_{3}$ and source $s_{1}$.

Below we present some basic notions from the graph theory which are used in the farther considerations [30-32]. A walk in a one-dimensional digraph $\mathscr{D}^{(1)}$ is a finite sequence of arcs in which every two vertices $v_{i}$ and $v_{j}$ are adjacent or identical. A walk in which all of the arcs are distinct is called a path. The path that goes through all vertices is called a finite path. If the initial and the terminal vertices of the path are the same, then the path is called a cycle. More information about digraph theory is given in [30], and the use of the digraph theory in positive system is given in $[33,34]$.

\section{Fractional order system}

\subsection{Model and representation}

The equation for a continuous-time dynamic system of the fractional-order can be written as follows:

$$
\begin{aligned}
& H\left(\mathfrak{D}^{\alpha_{0} \alpha_{1} \ldots \alpha_{m}}\right)\left(y_{1}, y_{2}, \ldots, y_{l}\right) \\
& \quad=G\left(\mathfrak{D}^{\beta_{0} \beta_{1} \ldots \beta_{n}}\right)\left(u_{1}, u_{2}, \ldots, y_{k}\right),
\end{aligned}
$$

where $y_{i}, u_{i}$ are the function of time and $H(\cdot), G(\cdot)$ are the combinations of the fractional-order derivative operator. If we describe the linear time-invariant single-variable case, we obtain the following equation:

$H\left(\mathfrak{D}^{\alpha_{0} \alpha_{1} \ldots \alpha_{m}}\right)\left(y_{t}\right)=G\left(\mathfrak{D}^{\beta_{0} \beta_{1} \ldots \beta_{n}}\right)\left(u_{t}\right)$,

with

$$
\begin{aligned}
& H\left(\mathfrak{D}^{\alpha_{0} \alpha_{1} \ldots \alpha_{n}}\right)=\sum_{k=0}^{n} a_{k} \mathfrak{D}^{\alpha_{k}}, \quad a_{k} \in \mathbb{R}, \\
& G\left(\mathfrak{D}^{\beta_{0} \beta_{1} \ldots \beta_{m}}\right)=\sum_{k=0}^{m} b_{k} \mathfrak{D}^{\beta_{k}}, \quad b_{k} \in \mathbb{R}
\end{aligned}
$$


or

$$
\begin{aligned}
& a_{n} \mathfrak{D}^{\alpha_{n}} y(t)+a_{n-1} \mathfrak{D}^{\alpha_{n-1}} y(t)+\cdots+a_{0} \mathfrak{D}^{\alpha_{0}} y(t) \\
& \quad=b_{m} \mathfrak{D}^{\beta_{m}} u(t)+b_{m-1} \mathfrak{D}^{\beta_{m-1}} u(t)+\cdots+b_{0} \mathfrak{D}^{\beta_{0}} u(t) .
\end{aligned}
$$

After aplying the Laplace transform to (1) with zero initial conditions, the input-output representation of a fractionalorder system can be obtained. The fractional-order system as the transfer function have the following form:

$G(s)=\frac{b_{m} s^{\beta_{m}}+b_{m-1} s^{\beta_{m-1}}+\cdots+b_{0} s^{\beta_{0}}}{a_{n} s^{\alpha_{n}}+a_{n-1} s^{\alpha_{n-1}}+\cdots+a_{0} s^{\alpha_{0}}}$.

In the case of a commensurate-order system, the continuoustime transfer function (2) is given in the following form $G(s)=\sum_{k=0}^{m} b_{k}\left(s^{\alpha}\right)^{k} / \sum_{k=0}^{n} a_{k}\left(s^{\alpha}\right)^{k}$.

The transfer function can be considered as a pseudorational function $H(\lambda)$ of the variable $\lambda=s^{\alpha}$ in the form:

$H(\lambda)=\frac{\sum_{k=0}^{m} b_{k} \lambda^{k}}{\sum_{k=0}^{n} a_{k} \lambda^{k}}=\frac{b_{0}+b_{1} \lambda+\cdots+b_{m} \lambda^{m}}{a_{0}+a_{1} \lambda+\cdots+a_{n} \lambda^{n}}$.

\subsection{State-space representation}

Let us consider the continuous-time fractional linear system described by state-space equations:

$$
\begin{aligned}
{ }_{0} \mathfrak{D}_{t}^{\alpha} x(t) & =\mathbf{A} x(t)+\mathbf{B} u(t), \quad 0<\alpha \leqslant 1 \\
y(t) & =\mathbf{C} x(t)+\mathbf{D} u(t)
\end{aligned}
$$

where $x(t) \in \mathbb{R}^{n}, u(t) \in \mathbb{R}^{m}, y(t) \in \mathbb{R}^{p}$ are the state, input and output vectors respectively and $\mathbf{A} \in \mathbb{R}^{n \times n}, \mathbf{B} \in \mathbb{R}^{n \times m}$, $\mathbf{C} \in \mathbb{R}^{p \times n}$ and $\mathbf{D} \in \mathbb{R}^{p \times m}$.

The following Caputo definition of the fractional derivative will be used:

${ }_{a}^{C} \mathfrak{D}_{t}^{\alpha}=\frac{d^{\alpha}}{d t^{\alpha}}=\frac{1}{\Gamma(n-\alpha)} \int_{a}^{t} \frac{f^{(n)}(\tau)}{(t-\tau)^{\alpha+1-n}} d \tau$,

where $\alpha \in \mathbb{R}$ is the order of a fractional derivative, $f^{(n)}(\tau)=$ $\frac{d^{n} f(\tau)}{d \tau^{n}}$ and $\Gamma(x)=\int_{0}^{\infty} e^{-t} t^{x-1} d t$ is the gamma function.

Theorem 1 The Laplace transform of the derivative-integral (5) has the form

$\mathscr{L}\left[\begin{array}{l}{ }_{0}^{C} \\ 0\end{array} \mathfrak{D}_{t}^{\alpha}\right]=s^{\alpha} F(s)-\sum_{k=1}^{n} s^{\alpha-k} f^{(k-1)}\left(0^{+}\right)$.

The proof of the Theorem 1 is given in [15].

Using the Laplace transform to (4), Theorem 1 and taking into account

$$
X(s)=\mathscr{L}[x(t)]=\int_{0}^{\infty} x(t) e^{-s t} d t,
$$

$\mathscr{L}\left[\mathfrak{D}^{\alpha} x(t)\right]=s^{\alpha} X(s)-s^{\alpha-1} x_{0}$

we obtain:

$X(s)=\left[\mathbf{I}_{n} s^{\alpha}-\mathbf{A}\right]^{-1}\left[s^{\alpha-1} x_{0}+\mathbf{B} U(s)\right]$,

$Y(s)=\mathbf{C X}(s)+\mathbf{D} U(s), U(s)=\mathscr{L}[u(t)]$.

Using (6) we can determine the transfer matrix of the system in the following form:

$\mathbf{T}(s)=\mathbf{C}\left[\mathbf{I}_{n} s^{\alpha}-\mathbf{A}\right]^{-1} \mathbf{B}+\mathbf{D}$.

Matrices A, B, C and $\mathbf{D}$ are called a realisation of the transfer matrix $\mathbf{T}(s) \in \mathbb{R}^{p \times m}(s)$ if they satisfy the equality (7). The realisation is called minimal if the dimension of the state matrix $\mathbf{A}$ is minimal among all possible realisations of $\mathbf{T}(s)$.

Remark 1 If we consider a positive one-dimensional continuous-time fractional linear system then Definition 1, Definition 2 and Definition 3 must be met.

Definition 1 The fractional system (4) is called the internally positive fractional system if and only if $x(t) \in \mathbb{R}_{+}^{n}$ and $y(t) \in \mathbb{R}_{+}^{p}$ for $t \geqslant 0$ for any initial conditions $x_{0} \in \mathbb{R}_{+}^{n}$ and all inputs $u(t) \in \mathbb{R}_{+}^{m}$ for $t \geqslant 0$.

Definition 2 A square real matrix $\mathbf{A}=\left[a_{i j}\right]$ is called the Metzler matrix if its off-diagonal entries are non-negative, i.e. $a_{i j} \geqslant 0$ for $i \neq j$.

Definition 3 The fractional system (4) is positive if and only if

$\mathbf{A} \in \mathbb{M}^{n \times n}, \mathbf{B} \in \mathbb{R}_{+}^{n \times m}, \mathbf{C} \in \mathbb{R}_{+}^{p \times n}, \mathbf{D} \in \mathbb{R}_{+}^{p \times m}$.

Matrices (8) are called a positive realisation of the transfer matrix $\mathbf{T}(s) \in \mathbb{R}_{+}^{p \times m}(s)$ if they satisfy the equality (7). The positive realisation is called minimal if the dimension of the state matrix $\mathbf{A}$ is minimal among all possible realisations of $\mathbf{T}(s)$.

\subsection{Problem formulation}

For a given transfer function (7), determine a minimal realisation (or minimal positive realisation) of the system (4) using the one-dimensional $\mathscr{D}^{(1)}$ digraphs theory. The dimension of the system must be the minimal among possible.

\section{Problem solution}

We can transfer matrix (7) present in the form (3). In this case, the transfer matrix is the function of the operator $\lambda=s^{\alpha}$

$\mathbf{T}(\lambda)=\mathbf{C}\left[\mathbf{I}_{n} \lambda-\mathbf{A}\right]^{-1} \mathbf{B}+\mathbf{D}=\frac{\mathbf{N}(\lambda)}{d(\lambda)}$ 


$$
=\left[\begin{array}{ccc}
T_{11}(\lambda) & \cdots & T_{1 m}(\lambda) \\
\vdots & \ddots & \vdots \\
T_{p 1}(\lambda) & \cdots & T_{p m}(\lambda)
\end{array}\right] \in \mathbb{R}^{p \times m}(\lambda),
$$

for

$T_{k l}(\lambda)_{\substack{k=1, \ldots, p \\ l=1, \ldots, m}}=\frac{b_{n}^{k l} \lambda^{n}+b_{n-1}^{k l} \lambda^{n-1}+\cdots+b_{1}^{k l} \lambda+b_{0}^{k l}}{\lambda^{n}+a_{n-1}^{k l} \lambda^{n-1}+\cdots+a_{1}^{k l} \lambda+a_{0}^{k l}}$.

From (9) we have

$\mathbf{D}=\lim _{\lambda \rightarrow \infty} \mathbf{T}(\lambda)$

since $\lim _{\lambda \rightarrow \infty}\left[\mathbf{I}_{n} \lambda-\mathbf{A}\right]=0$. The strictly proper transfer matrix is given by the equation: $\mathbf{T}_{s p}(\lambda)=\mathbf{C}[\mathbf{I} \lambda-\mathbf{A}]^{-1} \mathbf{B}$.

For single-input single-output (SISO) system the transfer function has the following form:

$T(\lambda)=\frac{b_{n} \lambda^{n}+b_{n-1} \lambda^{n-1}+\cdots+b_{1} \lambda+b_{0}}{\lambda^{n}+a_{n-1} \lambda^{n-1}+\cdots+a_{1} \lambda+a_{0}}$

and the strictly proper transfer function is given by the equation:

$$
\begin{aligned}
& T_{s p}(\lambda)=T(\lambda)-\mathbf{D}=\frac{\widetilde{n}(\lambda)}{d(\lambda)} \\
& \quad=\frac{\widetilde{b}_{n-1} \lambda^{n-1}+\widetilde{b}_{n-2} \lambda^{n-2}+\cdots+\widetilde{b}_{1} \lambda+\widetilde{b}_{0}}{\lambda^{n}+a_{n-1} \lambda^{n-1}+a_{n-2} \lambda^{n-2}+\cdots+a_{1} \lambda+a_{0}} .
\end{aligned}
$$

After multiplying the nominator and denominator of (11) by $\lambda^{-n}$, we obtain:

$$
\begin{aligned}
T_{s p}(\lambda) & =\frac{\widetilde{n}(\lambda)}{d(\lambda)} \\
& =\frac{\widetilde{b}_{n-1} \lambda^{-1}+\widetilde{b}_{n-2} \lambda^{-2}+\cdots+\widetilde{b}_{1} \lambda^{1-n}+\widetilde{b}_{0} \lambda^{-n}}{1+a_{n-1} \lambda^{-1}+a_{n-2} \lambda^{-2}+\cdots+a_{1} \lambda^{1-n}+a_{0} \lambda^{-n}}
\end{aligned}
$$

where:

$$
\begin{aligned}
& \widetilde{n}(\lambda)=\widetilde{b}_{n-1} \lambda^{-1}+\cdots+\widetilde{b}_{1} \lambda^{1-n}+\widetilde{b}_{0} \lambda^{-n} \\
& d(\lambda)=1+a_{n-1} \lambda^{-1}+\cdots+a_{1} \lambda^{1-n}+a_{0} \lambda^{-n}
\end{aligned}
$$

is the characteristic polynomial.

In the first step, the proposed method finds the state matrix A using decomposition of the characteristic polynomial $d(\lambda)$ into a set of simple monomials $d(\lambda)=1-M_{1}(\lambda)-M_{2}(\lambda)-$ $\ldots-M_{i}(\lambda)$, where $i$ is the number of monomials. For example, we can decompose the characteristic polynomial $d(\lambda)=1-\lambda^{-3}-2 \lambda^{-2}+4 \lambda^{-1}$ as a set of the simple monomials: $M_{1}=\lambda^{-3}, M_{2}=2 \lambda^{-2}$ and $M_{3}=-4 \lambda^{-1}$. For each simple monomial, we create a digraph representation. Then we can determine all possible characteristic polynomial realisations using all combinations of the digraph monomial representation.
Theorem 2 There exists a state matrix (or positive state matrix) $\mathbf{A}$ of the fractional continuous-time linear system (4) corresponding to the characteristic polynomial $d(\lambda)$ if:

i. the sets $\mathbb{D}_{1} \cdots \cap \cdots \mathbb{D}_{\text {mon }} \neq \emptyset$, mon $=1,2, \ldots$ corresponding to one-dimensional digraphs representing the monomials are not disjoint.

ii. the obtained digraph does not have any additional cycles.

Proof Condition (i): The sets $\mathbb{D}_{\text {mon }}$ mon $=1,2, \ldots$ are disjoint if $\mathbb{D}_{1} \cap \mathbb{D}_{2} \cdots \cap \cdots \mathbb{D}_{\text {mon-1 }} \cap \mathbb{D}_{\text {mon }}=\emptyset$. Then we have a digraph whose vertices can be divided into two disjoint sets (bipartite digraph). It means that we obtain an additional simple monomial in a characteristic polynomial $d(\lambda)$. In this situation, we obtain a new polynomial $\widetilde{d}(\lambda)(\widetilde{d}(\lambda) \neq d(\lambda))$. Condition (ii): Each monomial is represented by one cycle. If after combining all digraphs (each corresponding to one monomial) we obtain an additional cycle, this means that in the polynomial an additional simple monomial appears. We obtain a new polynomial $\bar{d}(\lambda)(\bar{d}(\lambda) \neq d(\lambda))$.

Remark 2 If we assume that realisation must be stable, then in Theorem 2 the following condition appears:

iii. the poles of the $\mathbf{T}(\lambda)$ are distinct real and negative i.e. $\lambda_{i}<0$ for $i=1,2 \ldots, n$ and $\lambda_{i} \neq \lambda_{j}$ for $i \neq j$.

Remark 3 If we assume that positive realisation must be stable, then in Theorem 2 the following conditions appear:

iv. $\mathbf{T}_{i}(\lambda)=\lim _{\lambda \rightarrow \lambda_{i}}=\frac{N\left(\lambda_{i}\right)}{\prod_{j=1, j \neq i}^{n}\left(\lambda_{i}-\lambda_{j}\right)} \in \mathbb{R}_{+}^{p \times m}$ for $i=1, \ldots, n$;

v. $\mathbf{T}(\infty) \in \mathbb{R}_{+}^{m \times m}$;

vi. the coefficients of the characteristic polynomial (13) are non-positive, i.e. $a_{k} \leqslant 0$ for $k=0,1, \ldots, n-2$.

The proof of the conditions (iii), (iv) and (v) is presented in paper [15]. From condition (iii), it follows that the characteristic polynomial has the form $d(\lambda)=\left(\lambda-\lambda_{1}\right)(\lambda-$ $\left.\lambda_{2}\right) \cdots\left(\lambda-\lambda_{n}\right)$, where $\lambda_{1}, \lambda_{2}, \ldots, \lambda_{n}$ are distinct real negative roots and coefficients are non-positive, i.e. $a_{k} \leqslant 0$ for $k=0,1, \ldots, n-2$.

The Algorithm 1 consists of two parts: the first, line 1-5, in which we construct all possible digraph representations for every monomial from a characteristic polynomial; the second, line $6-18$, in which we create all possible digraph structures using all combinations of the monomial digraph representations. The optimisation of the algorithm on which Algorithm 1 is based is presented in paper [35]. 
Let us assume that the matrix $\mathbf{B}$ and matrix $\mathbf{C}$ have the following form:

$\mathbf{B}=\left[\begin{array}{ccc}b_{11} & \ldots & b_{1, m} \\ b_{21} & \ldots & b_{2, m} \\ \vdots & \ddots & \vdots \\ b_{n, 1} & \ldots & b_{n, m}\end{array}\right], \quad \mathbf{C}=\left[\begin{array}{ccc}c_{11} & \ldots & c_{1, n} \\ c_{21} & \ldots & c_{2, n} \\ \vdots & \ddots & \vdots \\ c_{p, 1} & \ldots & c_{p, n}\end{array}\right]$

After inserting matrices (14) and determining state matrix $\mathbf{A}$ from the Algorithm 1 to the equality $\mathbf{T}_{s p}(\lambda)=$ $\mathbf{C}\left[\mathbf{I}_{n}-\mathbf{A} \lambda^{-1}\right]^{-1} \mathbf{B}$, we obtain a polynomial matrix $\overline{\mathbf{N}}(\lambda)$. After comparing variables with the same power of the polynomial matrix $\widetilde{\mathbf{N}}(\lambda)$ with the described polynomial matrix $\overline{\mathbf{N}}(\lambda)$, we obtain the set of the equations. After solving the equation, we obtain the matrices $\mathbf{B} \in \mathbb{R}^{n \times m}$ and $\mathbf{C} \in \mathbb{R}^{p \times n}$.
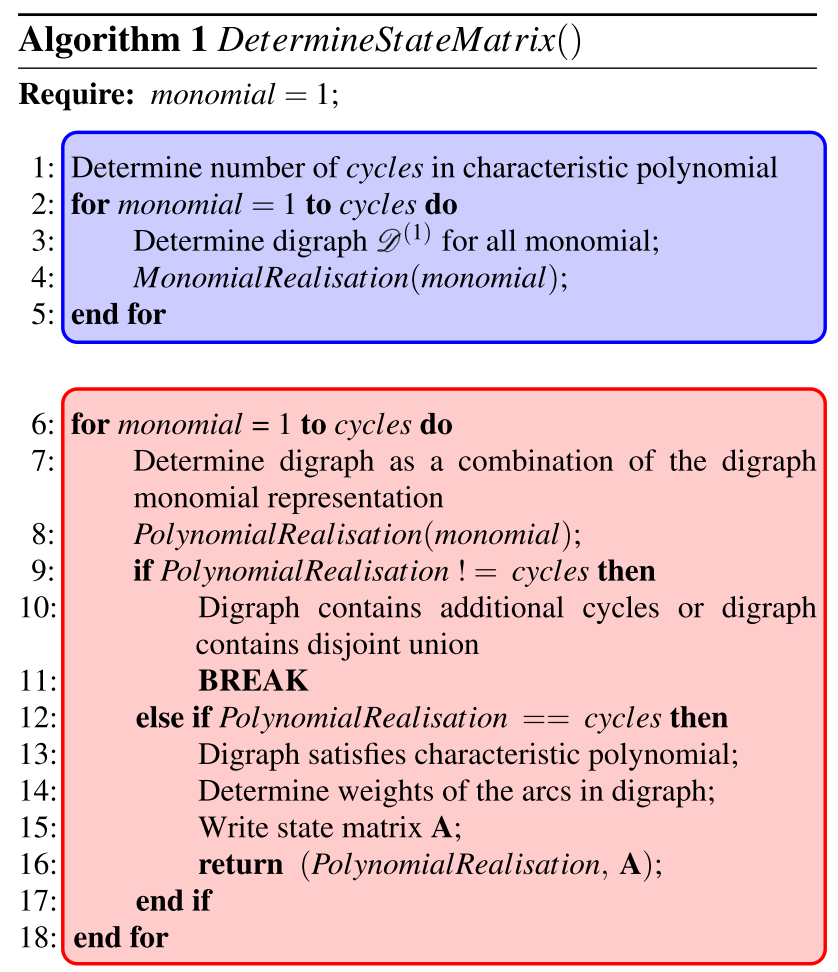

Remark 4 If we search for a positive realisation of the fractional continuous-time system, then the matrices $\mathbf{B} \in \mathbb{R}_{+}^{n \times m}$ and $\mathbf{C} \in \mathbb{R}_{+}^{p \times n}$ have non-negative entries.

In Fig. 2, we have presented how the algorithm for determination of the realisation of one-dimensional continuous-time fractional linear system works. In the first step, using (10), we must determine matrix D, then using Eq. (11) we determine a strictly proper transfer function. In the second step, using Algorithm 1, we determine the realisation of the state matrix A. Finally, we determine matrix $\mathbf{B}$ and matrix $\mathbf{C}$.

Thinking about realisation, if

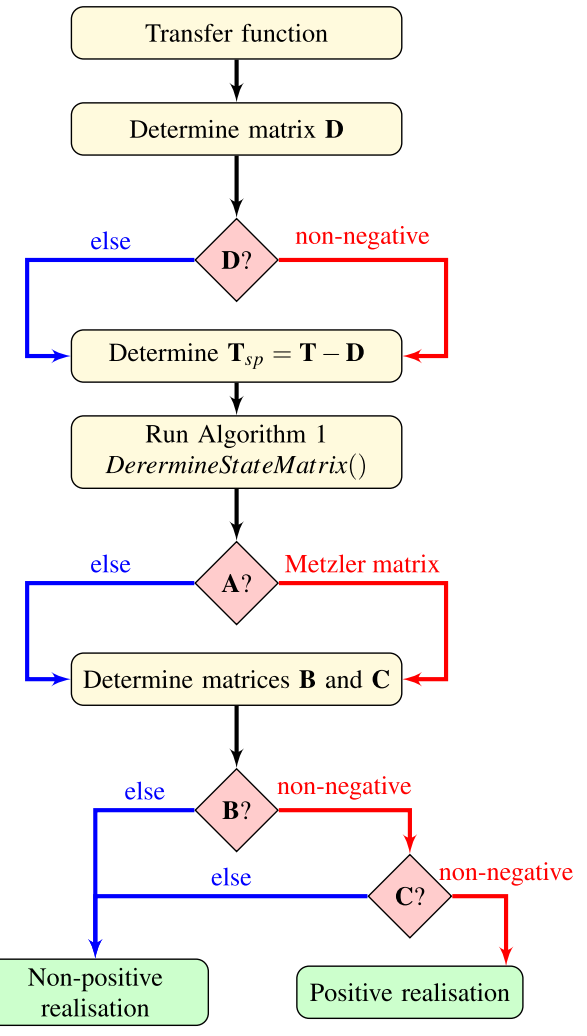

Fig. 2 Algorithm of the determination of the minimal realisation

- the matrix $\mathbf{A}$ is the Metzler matrix and matrices $\mathbf{B}, \mathbf{C}$ and D have non-negative entries, then the realisation is positive. If additionally positive realisation should be stable, then the condition (iii) from Remark 2 and conditions (iv), (v) and (vi) from Remark 3 must be met.

- Otherwise, the realisation is non-positive. If additionally realisation should be stable, then the condition (iii) from Remark 2 must be met.

\section{Numerical examples}

Consider the following three examples which show how to determine the minimal realisation of the continuous-time fractional system described by the Eq. (4).

\subsection{Example 1}

Find a positive minimal realisation of the transfer function

$T(s)=\frac{s^{3 \alpha}+0.7 s^{2 \alpha}+0.3 s^{\alpha}+0.1}{s^{3 \alpha}+0.6 s^{2 \alpha}-0.11 s^{\alpha}-0.06}$

for $0<\alpha<1$ 


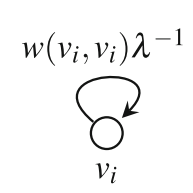

(a)

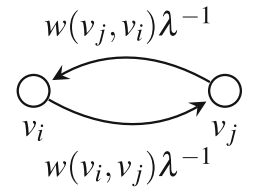

(b)

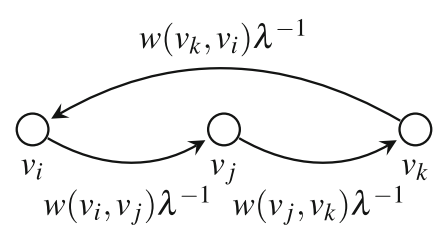

(c)

Fig. 3 One-dimensional digraphs corresponding to characteristic polynomial of the transfer function

Solution The transfer function (15) can be considered as a pseudo-rational function $T(\lambda)$ of the variable $\lambda=s^{\alpha}$ in the form:

$T(\lambda)=\frac{\lambda^{3}+0.7 \lambda^{2}+0.3 \lambda+0.1}{\lambda^{3}+0.6 \lambda^{2}-0.11 \lambda-0.06}$.

After multiplying the nominator and denominator of the transfer function (16) by $\lambda^{-3}$, we obtain:

$T(\lambda)=\frac{1+0.7 \lambda^{-1}+0.3 \lambda^{-2}+0.1 \lambda^{-3}}{1+0.6 \lambda^{-1}-0.11 \lambda^{-2}-0.06 \lambda^{-3}}$.

The matrix $\mathbf{D}$ for a given proper transfer matrix (17) can be computed using the formula:

$D=\lim _{\lambda \rightarrow \infty} \mathbf{T}(\lambda)=[1]$

From (11), (17) and (18) we have

$T_{s p}(\lambda)=\frac{0.1 \lambda^{-1}+0.41 \lambda^{-2}+0.16 \lambda^{-3}}{1+0.6 \lambda^{-1}-0.11 \lambda^{-2}-0.06 \lambda^{-3}}$.

In the first step, we write the following initial conditions: number of colours in digraphs: colour $=1$; monomials: $M_{1}=-0.6 \lambda^{-1}$ (Fig. 3a), $M_{2}=0.11 \lambda^{-2}$ (Fig. 3b), $M_{3}=$ $0.06 \lambda^{-3}$ (Fig. 3c).

Remark 5 In the further consideration, we will assume that $\mathscr{A}$-arcs are drawn by the solid line, and we assign the weight $w\left(v_{i}, v_{j}\right) \lambda^{-1}$.

Then, we determine all possible realisations of the monomial $M_{1}, M_{2}$ and $M_{3}$. We use the following notion: $M_{i} \mathscr{D}_{(j)}^{(n)}$ where: $M_{i}$ is the number of the monomial in a characteristic polynomial; $n$ is the digraph dimension and $j$ is the number of the monomial realisation.

$M_{1} \mathscr{D}_{(1)}^{(1)}=\left\{w\left(v_{1}, v_{1}\right)\right\}, M_{1} \mathscr{D}_{(2)}^{(1)}=\left\{w\left(v_{2}, v_{2}\right)\right\}$
$M_{1} \mathscr{D}_{(3)}^{(1)}=\left\{w\left(v_{3}, v_{3}\right)\right\}, M_{2} \mathscr{D}_{(1)}^{(1)}=\left\{w\left(v_{1}, 2\right), w\left(v_{2}, v_{1}\right)\right\}$,

$M_{2} \mathscr{D}_{(2)}^{(1)}=\left\{w\left(v_{2}, v_{3}\right), w\left(v_{3}, v_{2}\right)\right\}$

$M_{2} \mathscr{D}_{(3)}^{(1)}=\left\{w\left(v_{1}, v_{3}\right), w\left(v_{3}, v_{1}\right)\right\}$

$M_{3} \mathscr{D}_{(1)}^{(1)}=\left\{w\left(v_{1}, v_{2}\right), w\left(v_{2}, v_{3}\right), w\left(v_{3}, v_{1}\right)\right\}$.

Remark 6 Aside from a number of possible combinations of monomial realisations, there is a number of variants of possible ways to join sub-digraphs. The first monomial of the same size as the size of digraphs representing polynomial realisation will always have only 1 variant, as each other variant can be obtained by re enumerating vertices. For each other monomial realisation, there is a number of variants represented by the equation variants $=\frac{1}{n} \prod_{i=0}^{n-1}(m-i)$ where: $n$ is the size of sub-graph, and $m$ is the size of polynomial digraphs realisation.

In the described example, we have variants $=3 \cdot 3 \cdot 1=9$ possible minimal characteristic polynomial realisation consisting of three vertices. It should be noted that the potential and real solution does not contain realisations that we can receive by re-numbering the vertices in the digraphs. This operation is very simple and can be obtained by transposition of state matrix $\mathbf{A}$ after the algorithm is finished.

In Fig. 4, we have presented all possible realisations of the characteristic polynomial $d(\lambda)=1+0.6 \lambda^{-1}-0.11 \lambda^{-2}-$ $0.06 \lambda^{-3}$. The realisations which satisfy all conditions of the Theorem 2 are drawn in black, other realisations which do not satisfy any of the conditions of the Theorem 2 are drawn in red.

From all potential realisations, we choose the following two possible realisations.

The first realisation is presented in Fig. 5a. We check the conditions using Theorem 2 . To verify the first condition, we must compare sets (corresponding to representation of all simple monomial digraphs) $\mathbb{D}_{1} \cap \mathbb{D}_{2} \cap \mathbb{D}_{3}=\emptyset$. The described realisation does not satisfy Condition $(\mathbf{C 1})$. The realisation does not satisfy all conditions, and it is rejected.

The second realisation is presented in Fig. 5b. We check the conditions using Theorem 2 . To verify the first condition, we must compare sets (corresponding to representation of all simple monomial digraphs) $\mathbb{D}_{1} \cap \mathbb{D}_{2} \cap \mathbb{D}_{3}=\left\{v_{1}\right\}$. The described realisation satisfies Condition $(\mathbf{C 1})$. The obtained digraph does not appear in additional cycles. The Condition (C2) is satisfied. The realisation does satisfy all conditions, and it is correct.

From the obtained digraphs presented on Fig. 5b, we can write state matrix in the form:

$\mathbf{A}=\left[\begin{array}{ccc}w\left(v_{1}, v_{1}\right) & w\left(v_{2}, v_{1}\right) & w\left(v_{3}, v_{1}\right) \\ w\left(v_{1}, v_{2}\right) & 0 & 0 \\ 0 & w\left(v_{2}, v_{3}\right) & 0\end{array}\right]$ 
Fig. 4 All possible realisation of the characteristic polynomial $d(\lambda)$

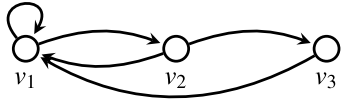

(a)

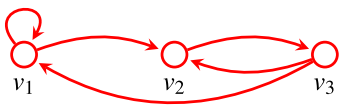

(d)

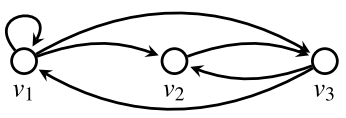

(g)

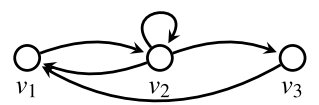

(b)

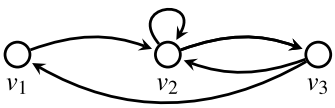

(e)

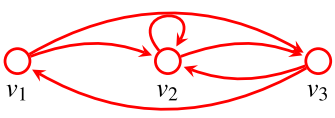

(h)

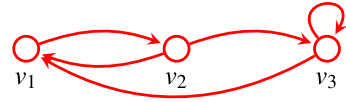

(c)

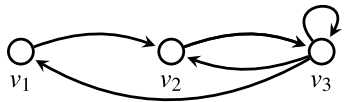

(f)

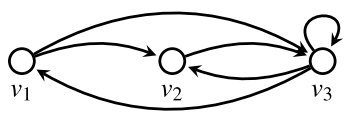

(i)
Fig. 5 Digraph representing minimal realisation of the characteristic polynomial $d(\lambda)$ : (a) not satisfying Theorem 2; (b) satisfying Theorem 2

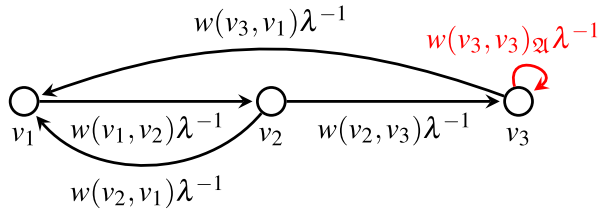

(a)

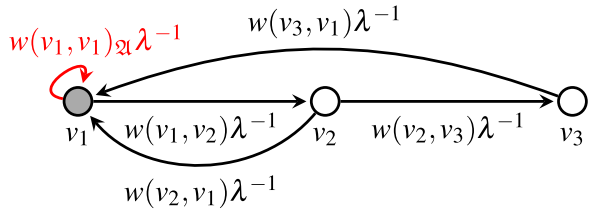

(b)

$$
=\left[\begin{array}{ccc}
-0.6 & 0.11 & 0.06 \\
1 & 0 & 0 \\
0 & 1 & 0
\end{array}\right] .
$$

After inserting matrices (14) and (19) to the equation $T_{s p}(\lambda)=\mathbf{C}\left[\mathbf{I}_{n}-\mathbf{A} \lambda^{-1}\right]^{-1} \mathbf{B}$ we obtain the polynomial $\bar{n}(\lambda)$. After the comparison of the coefficients of the same power $\lambda$ polynomials $\tilde{n}(\lambda)=\bar{n}(\lambda)$, we receive the set of the equations:

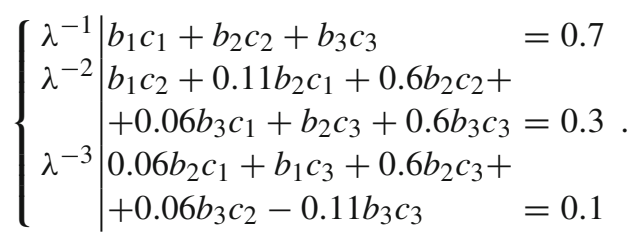

Solving them, we obtain the following matrices:

$$
\begin{aligned}
& \mathbf{B}=\left[\begin{array}{l}
b_{1} \\
b_{2} \\
b_{3}
\end{array}\right]=\left[\begin{array}{c}
0.1 \\
2.6667 \\
1.9450
\end{array}\right], \\
& \mathbf{C}=\left[\begin{array}{lll}
c_{1} & c_{2} & c_{3}
\end{array}\right]=\left[\begin{array}{lll}
1 & 0 & 0
\end{array}\right] .
\end{aligned}
$$

The desired positive realisation of the (15) is given by (18)(20).

\subsection{Example 2}

Find a positive minimal realisation of the transfer matrix

$\mathbf{T}(s)=\left[\begin{array}{cc}\frac{s^{a}+2.1}{s^{a}+0.1} & \frac{2 s^{a}+1.4}{s^{a}+0.2} \\ \frac{1}{s^{a}+0.2} & \frac{s^{a}+1.3}{s^{a}+0.3}\end{array}\right]$

with the order $\alpha=a, 0<a<1$.

Solution The transfer function (21) can be considered as a pseudo-rational transfer matrix $\mathbf{T}(\lambda)$ of the variable $\lambda=s^{a}$ in the form:

$\mathbf{T}(\lambda)=\left[\begin{array}{ll}\frac{\lambda+2.1}{\lambda+0.1} & \frac{2 \lambda+1.4}{\lambda+0.2} \\ \frac{1}{\lambda+0.2} & \frac{\lambda+1.3}{\lambda+0.3}\end{array}\right]$.

After multiplying the nominator and denominator of the transfer function (22) by $\lambda^{-1}$, we obtain:

$\mathbf{T}(\lambda)=\left[\begin{array}{cc}\frac{1+2.1 \lambda^{-1}}{1+0.1 \lambda^{-1}} & \frac{2+1.4 \lambda^{-1}}{1+0.2 \lambda^{-1}} \\ \frac{\lambda^{-1}}{1+0.2 \lambda^{-1}} & \frac{1+1.3 \lambda^{-1}}{1+0.3 \lambda^{-1}}\end{array}\right]$.

The matrix $\mathbf{D}$ for a given proper transfer matrix (23) can be computed using the formula:

$\mathbf{D}=\lim _{\lambda \rightarrow \infty} \mathbf{T}(\lambda)=\left[\begin{array}{ll}1 & 2 \\ 0 & 1\end{array}\right]$ 


$$
w\left(v_{i}, v_{i}\right) \lambda^{-1} \bigodot_{v_{i}}^{\gtrless}
$$

Fig. 6 One-dimensional digraphs corresponding to characteristic polynomial $d_{i}(\lambda), i=1, \ldots, 4$

From (11), (23) and (24) we have

$$
\mathbf{T}_{s p}(\lambda)=\left[\begin{array}{ll}
\frac{2 \lambda^{-1}}{1+0.1 \lambda^{-1}} & \frac{\lambda^{-1}}{1+0.2 \lambda^{-1}} \\
\frac{\lambda^{-1}}{1+0.2 \lambda^{-1}} & \frac{\lambda^{-1}}{1+0.3 \lambda^{-1}}
\end{array}\right] .
$$

The problem of determining of the minimal characteristic polynomial realisation can be divided into four smaller subproblems. In the first step, we write the following initial conditions: number of colours in digraphs: colour $=1$; characteristic polynomial: $d_{1}(\lambda)=1+0.1 \lambda^{-1}, d_{2}(\lambda)=d_{3}(\lambda)=$ $1+0.2 \lambda^{-1}, d_{4}(\lambda)=1+0.3 \lambda^{-1}$. It should be noted that characteristic polynomial $d_{i}(\lambda), i=1, \ldots, 4$, consists of one monomial $M_{i}: M_{1}=0.1 \lambda^{-1}$ (Fig. 6), $M_{2}=M_{3}=0.2 \lambda^{-1}$ (Fig. 6), and $M_{4}=0.3 \lambda^{-1}$ (Fig. 6).

Since the polynomial consists of one monomial there is only one of its realisations presented in Fig. 6. The realisation meets conditions (i) and (ii) of the Theorem 2. From the obtained digraphs, we can write state matrix in the form:

$\mathbf{A}=\left[\begin{array}{cccc}-0.1 & 0 & 0 & 0 \\ 0 & -02 & 0 & 0 \\ 0 & 0 & -0.2 & 0 \\ 0 & 0 & 0 & -0.3\end{array}\right]$

After inserting matrices (14) and (25) to the equation $\mathbf{T}_{s p}(\lambda)=\mathbf{C}\left[\mathbf{I}_{n}-\mathbf{A} \lambda^{-1}\right]^{-1} \mathbf{B}$, we obtain the polynomial matrix $\overline{\mathbf{N}}(\lambda)$. After the comparison of the coefficients of the same power $\lambda$ polynomials $\widetilde{n}_{p, m}(\lambda)=\bar{n}_{p, m}(\lambda)$, we receive the set of the equations. After solving them, we obtain the following matrices:

$\mathbf{B}=\left[\begin{array}{ll}2 & 0 \\ 0 & 1 \\ 1 & 0 \\ 0 & 1\end{array}\right], \mathbf{C}=\left[\begin{array}{llll}1 & 1 & 0 & 0 \\ 0 & 0 & 1 & 1\end{array}\right]$

The desired positive realisation of the (21) is given by (25), (26) and (24).

\subsection{Example 3}

Find positive and non-positive minimal stable realisations of the transfer matrix

$T_{s p}(s)=\frac{s^{2 \alpha}+6 s^{\alpha}+8}{s^{3 \alpha}+9 s^{2 \alpha}+23 s^{\alpha}+15}$ with the order $0<\alpha<1$.

Solution The transfer function (27) can be considered as a pseudo-rational function $T(\lambda)$ of the variable $\lambda=s^{\alpha}$ in the form:

$T_{s p}(\lambda)=\frac{\lambda^{2}+6 \lambda+8}{\lambda^{3}+9 \lambda^{2}+23 \lambda+15}$.

Case 1: Stable non-positive minimal realisation In this case, we have $d(\lambda)=(\lambda+1)(\lambda+3)(\lambda+5), \lambda_{1}=-1$, $\lambda_{2}=-3, \lambda_{3}=-5$ and the condition (iii) of Remark 2 is met.

After multiplying the nominator and denominator of the transfer function (28) by $\lambda^{-3}$, we obtain:

$T_{s p}(\lambda)=\frac{\lambda^{-1}+6 \lambda^{-2}+8 \lambda^{-3}}{1+9 \lambda^{-1}+23 \lambda^{-2}+15 \lambda^{-3}}$.

In the first step, we write the following initial conditions: number of colours in digraphs: colour $=1$; monomials: $M_{1}=-9 \lambda^{-1}$ (Fig. 3a), $M_{2}=-23 \lambda^{-2}$ (Fig. 3b), $M_{3}=$ $-15 \lambda^{-3}$ (Fig. 3c).

From all potential realisations, we choose the following possible realisations presented in Fig. 5b. As shown in Example 2, the digraph structure does satisfy all conditions of the Theorem 2, and it is correct.

From the obtained digraphs presented in Fig. 5b, we can write a state matrix in the form:

$$
\begin{aligned}
\mathbf{A} & =\left[\begin{array}{ccc}
w\left(v_{1}, v_{1}\right) & w\left(v_{2}, v_{1}\right) & w\left(v_{3}, v_{1}\right) \\
w\left(v_{1}, v_{2}\right) & 0 & 0 \\
0 & w\left(v_{2}, v_{3}\right) & 0
\end{array}\right] \\
& =\left[\begin{array}{ccc}
-9 & -23 & -15 \\
1 & 0 & 0 \\
0 & 1 & 0
\end{array}\right]
\end{aligned}
$$

Matrix $\mathbf{A}$ is not a Metzler matrix and its realisation is nonpositive. In the next step, we must solve the following set of the equations:

$$
\left\{\begin{array}{l|l}
\lambda^{-1} & \begin{array}{ll}
b_{1} c_{1}+b_{2} c_{2}+b_{3} c_{3} & =1 \\
\lambda^{-2} & b_{1} c_{2}-23 b_{2} c_{1}+9 b_{2} c_{2}-15 b_{3} c_{1}+ \\
+b_{2} c_{3}+9 b_{3} c_{3} & =6 \\
\lambda^{-3} & \begin{array}{l}
15 b_{2} c_{1}+b_{1} c_{3}+9 b_{2} c_{3}-15 b_{3} c_{2}+ \\
+23 b_{3} c_{3}
\end{array}
\end{array}
\end{array}\right.
$$

After solving them, we obtain the following matrices:

$$
\begin{aligned}
& \mathbf{B}=\left[\begin{array}{l}
b_{1} \\
b_{2} \\
b_{3}
\end{array}\right]=\left[\begin{array}{c}
1 / c_{1} \\
-8 / 15 c_{1} \\
94 / 225 c_{1}
\end{array}\right], \\
& \mathbf{C}=\left[\begin{array}{lll}
c_{1} & c_{2} & c_{3}
\end{array}\right]=\left[\begin{array}{lll}
c_{1} & 0 & 0
\end{array}\right] .
\end{aligned}
$$


Fig. 7 Step response of the system (27) for $\alpha=\{0.3 ; 0.5 ; 0.7 ; 0.9\}$

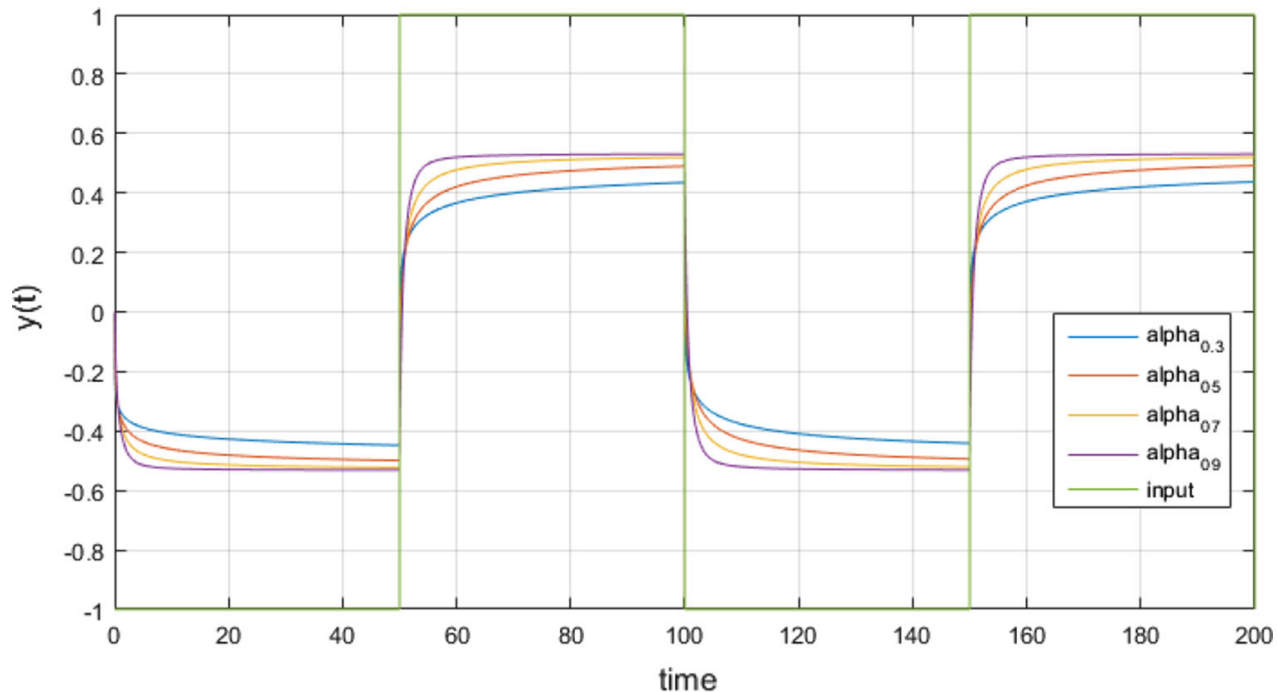

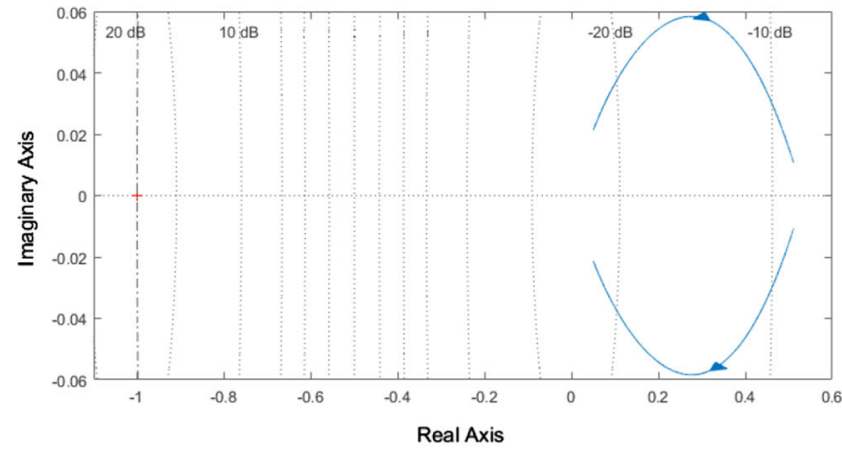

(a)

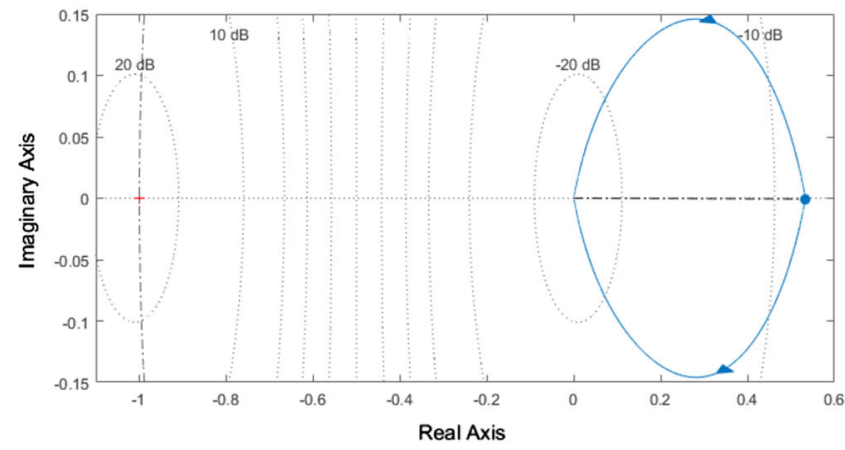

(c)

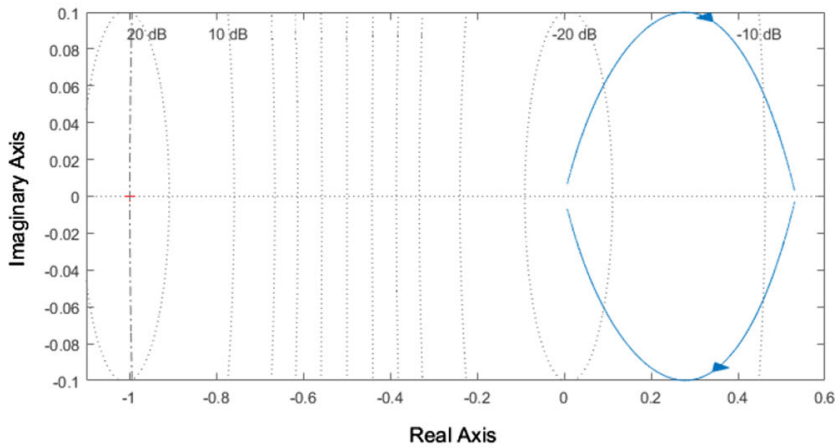

(b)

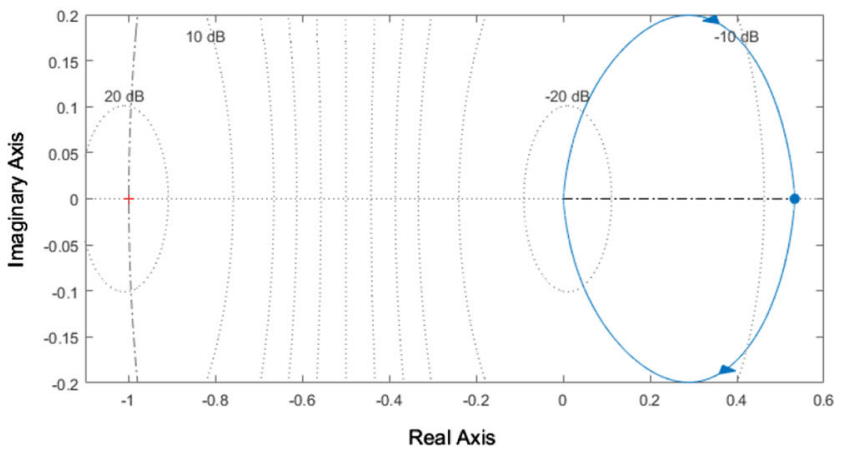

(d)

Fig. 8 Nyquist characteristic of the system (27) for: (a) $\alpha=0.3$; (b) $\alpha=0.5 ;$ (c) $\alpha=0.7$; (d) $\alpha=0.9$

The desired non-positive realisation of the (27) is given by (29) and (30).

The obtained realisation is also stable, as can be seen in Fig. 7, showing the step response of the system for $\alpha$ equal to: $0.3,0.5,0.7$ and 0.9 . In Fig. 8a, showing the Nyquist characteristic for: $\alpha=0.3$ (Fig. 8a); $\alpha=0.5$ (Fig. 8b); $\alpha=0.7$ (Fig. 8c); $\alpha=0.9$ (Fig. 8d). Simulations were performed using Matlab toolbox FOMCON (Fractional-order Modelling and Control) presented in the paper [36,37].
Case 2: Stable positive minimal realisation In the considered case, a realisation must be positive and stable. As shown in the first case, the condition (iii) of the Remark 2 is met $\left(\lambda_{1}=-1, \lambda_{2}=-3, \lambda_{3}=-5\right)$. In the next step, we check the stability of the positive system. After using condition (iv) of the Remark 3 and (28), we obtain:

$\mathbf{T}_{1}=\left.\lim _{\lambda \rightarrow \lambda_{1}} \frac{\lambda^{2}+6 \lambda+8}{\left(\lambda_{1}-\lambda_{3}\right)\left(\lambda_{1}-\lambda_{3}\right)}\right|_{\mid \lambda_{1}=-1}=\frac{3}{8} \in \mathbb{R}_{+}$, 


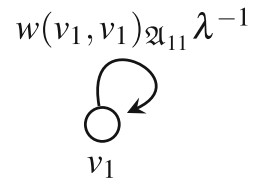

(a)

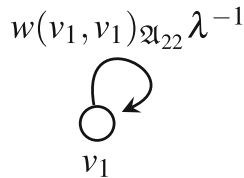

(b)

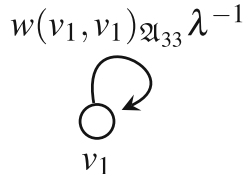

(c)
Fig. 9 One-dimensional digraphs corresponding to simple polynomial: (a) $d_{11}(\lambda) ;(\mathbf{b}) d_{22}(\lambda) ;(\mathbf{c}) d_{33}(\lambda)$

$$
\begin{aligned}
& \mathbf{T}_{2}=\left.\lim _{\lambda \rightarrow \lambda_{2}} \frac{\lambda^{2}+6 \lambda+8}{\left(\lambda_{2}-\lambda_{1}\right)\left(\lambda_{2}-\lambda_{3}\right)}\right|_{\mid \lambda_{2}=-3}=\frac{1}{4} \in \mathbb{R}_{+}, \\
& \mathbf{T}_{3}=\left.\lim _{\lambda \rightarrow \lambda_{3}} \frac{\lambda^{2}+6 \lambda+8}{\left(\lambda_{3}-\lambda_{1}\right)\left(\lambda_{3}-\lambda_{2}\right)}\right|_{\mid \lambda_{2}=-5}=\frac{3}{8} \in \mathbb{R}_{+} .
\end{aligned}
$$

Conditions (v) and (vi) of the Remark 3 are also met.

In the next step, we decompose a characteristic polynomial into a set of simple polynomials

$$
\begin{aligned}
d(\lambda) & =1+9 \lambda^{-1}+23 \lambda^{-2}+15 \lambda^{-3} \\
& =\underbrace{\left(1+\lambda^{-1}\right)}_{d_{11}(\lambda)} \underbrace{\left(1+3 \lambda^{-1}\right)}_{d_{22}(\lambda)} \underbrace{\left(1+5 \lambda^{-1}\right)}_{d_{33}(\lambda)} .
\end{aligned}
$$

Therefore, for each simple monomial we write initial conditions. For a simple polynomial $d_{11}(\lambda)$, we have the following conditions: number of vertices in digraph: vertices $=$ 1; possible weights from which we will build digraphs: $\left(v_{i}, v_{j}\right)_{\mathfrak{A}_{11}} \lambda^{-1}$; monomials: $M_{1}=1+\lambda^{-1}$.

Then, we determine all possible realisations of the simple polynomial $d_{11}(\lambda)$. In the considered example, we have only one realisation presented in Fig. 9a. The realisation meets conditions (i) and (ii) of the Theorem 2, and it is correct. In the same way, we can determine realisations of the simple polynomial $d_{22}(\lambda)$ presented in Fig. $9 \mathrm{~b}$ and $d_{33}(\lambda)$ presented in Fig. 9c. From the obtained digraphs, we can write the state matrix $\mathbf{A}$ in the form:

$$
\begin{aligned}
\mathbf{A} & =\left[\begin{array}{ccc}
\mathbf{A}_{11} & 0 & 0 \\
0 & \mathbf{A}_{22} & 0 \\
0 & 0 & \mathbf{A}_{33}
\end{array}\right] \\
& =\left[\begin{array}{ccc}
w\left(v_{1}, v_{1}\right)_{\mathfrak{A}_{11}} & 0 & 0 \\
0 & w\left(v_{1}, v_{1}\right)_{\mathfrak{A}_{22}} & 0 \\
0 & 0 & w\left(v_{1}, v_{1}\right)_{\mathfrak{A}_{33}}
\end{array}\right] \\
& =\left[\begin{array}{ccc}
-1 & 0 & 0 \\
0 & -3 & 0 \\
0 & 0 & -5
\end{array}\right] \in \mathbb{M} .
\end{aligned}
$$

Finally, we must solve the following set of the equations:

$$
\left\{\begin{array}{l|ll}
\lambda^{-1} & b_{1} c_{1}+b_{2} c_{2}+b_{3} c_{3} & =1 \\
\lambda^{-2} & 8 b_{1} c_{1}+6 b_{2} c_{2}+4 b_{3} c_{3} & =6 \\
\lambda^{-3} & 15 b_{1} c_{1}+5 b_{2} c_{2}+3 b_{3} c_{3} & =8
\end{array} .\right.
$$

After solving them, we obtain the following matrices:

$$
\begin{aligned}
& \mathbf{B}=\left[\begin{array}{l}
b_{1} \\
b_{2} \\
b_{3}
\end{array}\right]=\left[\begin{array}{c}
0.375 / c_{1} \\
0.25 / c_{2} \\
0.375 / c_{3}
\end{array}\right], \\
& \mathbf{C}=\left[\begin{array}{ll}
c_{1} c_{2} c_{3}
\end{array}\right]=\left[\begin{array}{lll}
c_{1} c_{2} c_{3}
\end{array}\right] .
\end{aligned}
$$

The desired positive realisation of the (27) is given by (31) and (33) for $c_{1}>0, c_{2}>0$ and $c_{3}>0$.

The obtained realisation is also stable, as can be seen in Figs. 7 and 8(a)-(d).

\section{Concluding remarks}

The paper presents a method, based on the one-dimensional digraphs theory, for finding the complete set of one-dimensional characteristic polynomial realisations, that can be used to solve the minimal positive realisation problem of a one-dimensional continuous-time fractional system which includes single-input and single-output (SISO) and multi-input as well as multi-output (MIMO). The difference between the algorithm proposed in this paper and currently used methods based on canonical forms of the system (i.e. constant matrix forms) is the creation not one (or few) minimal realisations, but a set of every possible minimal realisation.

Further work includes extension of the algorithm to find all possible solutions, solving the realisation problem, reachability and controllability of systems using the fast graph-based method and extension of the algorithm to find the class of electrical circuits corresponding to the transmission matrix. There is also a very difficult open problem of the analysis of systems dynamics for realisations on a different number of nodes in digraphs.

Open Access This article is distributed under the terms of the Creative Commons Attribution 4.0 International License (http://creativecomm ons.org/licenses/by/4.0/), which permits unrestricted use, distribution, and reproduction in any medium, provided you give appropriate credit to the original author(s) and the source, provide a link to the Creative Commons license, and indicate if changes were made.

\section{References}

1. Benvenuti L, Farina L (2004) A tutorial on the positive realization problem. IEEE Trans Autom Control 49(5):651-664

2. Farina L, Rinaldi S (2000) Positive linear systems: theory and applications. Series on pure and applied mathematics. WileyInterscience, New York

3. Kaczorek T (1985) Two-dimensional linear systems. Springer, London

4. Kaczorek T (2001) Positive 1D and 2D systems. Springer, London 
5. Kaczorek T (2007) Polynomial and rational matrices. Springer, London

6. Luenberger DG (1979) Introduction to dynamic systems: theory, models, and applications, chap. positive linear systems. Wiley, New York

7. Hryniów K, Markowski KA (2014) Parallel digraphs-building algorithm for polynomial realisations. In: Proceedings of 2014 15th international Carpathian control conference (ICCC), pp 174-179. doi:10.1109/CarpathianCC.2014.6843592

8. Hryniów K, Markowski KA (2014) Reachability index calculation by parallel digraphs-building. In: 19th international conference on methods and models in automation and robotics (MMAR), Miedzyzdroje, Poland, September 2-5, 2014, pp 808-813. doi:10. 1109/MMAR.2014.6957460

9. Markowski KA, Hryniów K (2015) Solving minimal realisation problem of positive two-dimensional hybrid systems in the form of second fornasini-marchesini model. In: Proceedings of 2nd IEEE international conference on cybernetics, CD-ROM, Gdynia, Poland, June 24-26, 2015, pp 172-177. doi:10.1109/CYBConf. 2015.7175927

10. Markowski KA (2015) New digraphs-building algorithm for minimal realisations of one-dimensional fractional positive systems with delays. In: Proceedings of 19th international conference on system theory, control and computing, pp 545-550

11. Markowski KA (2015) Positive stable realisation of fractional electrical circuits consisting of $\mathrm{n}$ subsystem. J Phys Conf Ser 659(1):012041. http://stacks.iop.org/1742-6596/659/ $\mathrm{i}=1 / \mathrm{a}=012041$

12. Markowski KA, Hryniów K (2015) Digraphs minimal positive stable realisations for fractional one-dimensional systems. In: Proceedings of 7th conference on non-integer order calculus and its applications, pp 105-118. doi:10.1007/978-3-319-23039-9_9

13. Nishimoto K (1984) Fractional calculus. Decartess Press, Koriama

14. Das S (2011) Functional fractional calculus. Springer, New York. doi:10.1007/978-3-642-20545-3

15. Kaczorek T, Sajewski L (2014) The realization problem for positive and fractional systems. Springer, Berlin. doi:10.1007/ 978-3-319-04834-5

16. Miller K, Ross B (1993) An introduction to the fractional calculus and fractional differenctial equations. Willeys, New York

17. Ortigueira MD (2011) Fractional calculus for scientists and engineers. Academic Press, Netherlands. doi:10.1007/ 978-94-007-0747-4

18. Podlubny I (1999) Fractional differential equations. Academic Press, San Diego

19. Lopes AM, Machado JAT (2015) Visualizing control systems performance: a fractional perspective. Adv Mech Eng 7(12): 1687814015619831

20. Machado J, Lopes AM (2015) Fractional state space analysis of temperature time series. Fract Calc Appl Anal 18(6):1518-1536

21. Machado J, Mata ME, Lopes AM (2015) Fractional state space analysis of economic systems. Entropy 17(8):5402-5421

22. Magin R, Ortigueira MD, Podlubny I, Trujillo J (2011) On the fractional signals and systems. Signal Process 91(3):350-371
23. Martynyuk V, Ortigueira M (2015) Fractional model of an electrochemical capacitor. Signal Process 107:355-360

24. Ortigueira MD, Rivero M, Trujillo JJ (2015) Steady-state response of constant coefficient discrete-time differential systems. J King Saud Univ Sci

25. Ortigueira MD, Simos TE, Psihoyios G, Tsitouras C, Anastassi Z (2012) Two-sided discrete fractional derivatives and systems. AIP Conf Proc Am Inst Phys 1479(1):1416

26. Petras I, Sierociuk D, Podlubny I (2012) Identification of parameters of a half-order system. IEEE Trans Signal Process 60(10):5561-5566

27. Podlubny I, Skovranek T, Datsko B (2014) Recent advances in numerical methods for partial fractional differential equations. In: Control conference (ICCC), 2014 15th international Carpathian. IEEE, pp 454-457

28. Berman A, Neumann M, Stern RJ (1989) Nonnegative matrices in dynamic systems. Wiley, New York

29. Horn RA, Johnson CR (1991) Topics in matrix analysis. Cambridge University Press, Cambridge

30. Bang-Jensen J, Gutin G (2009) Digraphs: theory, algorithms and applications. Springer, London

31. Godsil C, Royle G (2001) Algebraic graph theory. Springer, Berlin

32. Wallis WD (2007) A beginner's guide to graph theory. Biiokhäuser, Basel

33. Fornasini E, Valcher ME (1997) Directed graphs, 2D state models, and characteristic polynomials of irreducible matrix pairs. Linear Algebra Appl 263:275-310

34. Fornasini E, Valcher ME (2005) Controllability and reachability of 2D positive systems: a graph theoretic approach. IEEE Trans Circuits Syst I(52):576-585

35. Hryniów K, Markowski K (2015) Optimisation of digraphs-based realisations for polynomials of one and two variables. In: Szewczyk R, Zieliński C, Kaliczyńska M (eds) Progress in automation, robotics and measuring techniques, advances in intelligent systems and computing, vol 350. Springer, pp 73-83. doi:10.1007/ 978-3-319-15796-2_8

36. Tepljakov A, Petlenkov E, Belikov J (2011) FOMCON: a MATLAB toolbox for fractional-order system identification and control. Int J Microelectron Comput Sci 2(2):51-62

37. Tepljakov A, Petlenkov E, Belikov J (2011) FOMCON: fractional order modeling and control toolbox for MATLAB. In: The 18th international conference mixed design of integrated circuits and systems, Gliwice, Poland, pp 684-689 